\title{
浅谈多波束声呐系统的水深测量技术研究
}

任皓 张少飞

DOI:10.32629/hwr.v4i1.2696

[摘 要] 多波束测深系统组成复杂,集成度高、数据处理环节多、数据同步性要求严格,对操作人员的要求也比较高。所以要获得理想的测试 效果,必须要对多波束测深系统有全面的认识。同时多波束测深系统进行水深地形测量时,会受到各种影响,本文中对此进行了简单的介绍,希望 可以对多波束测深系统的水深地形测量作业提供一定的参考指导作用。

[关键词] 多波束测深系统; 航道炸礁; 质量检验; 应用研究

测深准确度和有效条带宽度是直观衡量多波束声纳计量性能的主要 几何指标, 影响水下地形测量成果质量和作业效率。目前我国能开展多波 束声纳测深性能计量测试的实验室屈指可数, 原因在于多波束声纳计量测 试需要大型试验水池的支撑, 由于受限于室内水池尺寸, 测深范围一般不 会超过 $50 \mathrm{~m}$, 远不能满足浅水型多波束声纳的计量需求。潮汐、涌浪、剖 面声速、海底地形变化等外场环境影响因素, 给海上自校或比测结果引入 较大误差。

\section{1 多波束声呐系统工作原理}

多波束声呐测深系统的基本原理, 就是在同时获得了多个波束在水底 的传递过程当中, 结合了声速在水底下的传播原理, 使用斯涅耳法则来实 施声音线路的跟踪, 通过这种方式来计算声波在船体运行坐标下面的水体 深度, 然后再使用实施性的三位姿态数据分析, 以及通过全球卫星导航系 统的定位, 将船体坐标系下面的水体深度转换到对应的坐标系下面, 最终 得到了声波对应的水体深度和相应的坐标。多波束声呐系统一次性发射与 接收声音脉冲的时候, 可以形成上百个声音波束, 也就是可以得到上百个 水深测量数据, 极大的提升了水深测量工作的工作效率。

\section{2 多波束水下地形扫测}

本次项目扫测采用的多波束测深系统是由丹麦RESON公司生产的 SeaBat7125SV2型高分辨率多波束声呐系统。本次扫测项目多波束采用船 体井式固定安装。数据采集利用 $7 \mathrm{KContro1}$ 控制软件与PDS2000数据采集软 件相结合的方式进行。采用7KControl控制软件对各个测量传感器进行控 制; PDS2000数据采集软件用于数据采集以及数据显示。扫测时, 首先在 PDS2000中建立扫测项目。数据采集的具体过程如下。(1) 在新建扫测项目 前要利用能够控制到该测区范围的控制点计算出测区的七参数。(2) 在已 有的测区范围图上用直线段取出范围线并保存为 $d x f$ 格式。(3) 新建扫测项 目。(4) 插入 $7 \mathrm{~K}$ 控制软件狗, 打开 $7 \mathrm{~K}$ 控制软件, 选择适合本次测区水深的 $400 \mathrm{kHz}$ 频率, 以自动模式开始数据采集。(5) 打开PDS2000软件点击 Realtime, 显示一些常见的便于操作者和测船驾驶员观看的界面。

\section{3 多波束数据处理与成图}

3. 1 数据处理

(1) 新建船型文件, 船型文件的日期要在本次扫测开始前的某个日期, 船型文件主要是用于把多波束系统指出各传感器的空间参考位置以及各 传感器的安装偏转角; (2) 新建工程, 工程日期最好是测量当天日期; (3) 导入当天所测的测线文件并打开新建的工程; (4) 添加当天所测区域内的 潮位文件和剖面声速文件并进行数据融合; (5) 选中所导入的一条测线文 件截取它的一条狭小的剖面在各个视图下进行条带编辑, 以便剔除跳点; (6) 新建表单文件, 表单文件可以新建多个, 以便得到想要的某个区域内的 水深数据;（7) 新建基础曲面并刷新; (8) 选一个狭小的剖面在平面和三维 视图下进行分块编辑, 以便剔除跳点; (9) 把一些非常小没有扫测到的区域
利用内插的方法来获得水深数据; (10) 数据输出, 按照本次扫测的有关技 术要求, 输出格网大小为 $1 \mathrm{~m}$ 的水下地形数据。

3. 2 扫描声呐图像处理技术

作为潜艇开发的重要基础装备, 扫描声纳可以实现水下高分辨率成像, 直接影响海底探测的科学性和有效性。扫描声呐数据处理主要包括降噪和 坡度校正两个方面。距离成像、侧扫描声纳工作当传感器高从海底和系统 范围可达 $1: 10$ 的比例, 所以目标中的形象有严重的节略, 褶皱的面具和顶 点位移的几何失真, 更基于声线跟踪法用于消除直线距离函数; 侧扫声纳 系统的回声在一段时间内是水下声波的矢量, 它包含了各种噪声, 会使声 波被误判。因此, 必须处理扫描声纳数据的噪声。

\section{4 成果质量检验}

4.1扫床方式

目前内河航道整治硬式扫床方式多采用横式和顺式两种。根据水位情 况及本工程特点, 本工程计划采取大型工作船进行横式扫床。在前期水深 测量确认达到设计标高后进行。具体以工程船作为扫床平台, 在杆上设置 扫床架进行横式扫床。工程船由两根主缆以及左右 4 根横移钢缆固定船位, 通过左右横移钢缆的收放横向移动船位, 达到扫床目的。

4. 2 扫床技术措施

(1) 扫床架采用坚杆、横杆和斜支杆组装。(2) 扫床架中部多增加一根 横加强杆, 提高了入水部分扫架的整体强度。(3) 扫床底扫杆平行重叠一根 和扫床架杆全焊接方式连接, 防止扫杆因水流影响和拉绳受力变形。(4) 增加上游方向第二层提头绳 1 根、左右拉绳 4 根, 防止扫架左右摆动。(5) 为了保证扫床质量, 在扫床架横扫杆上设置一根细钢缆, 用于检测坚直扫 杆的变形。(6) 随时对扫测船左右侧针的走向进行调整, 使扫测船处于最佳 扫测模式, 确保扫床范围全覆盖和扫床安全。

\section{5 精度评价与对比}

5.1 扫测宽度及分辨率

目前我单位正在使用的SeaBat7125型多波束系统, 一次发射 512 个声 波波束, 等距模式下波束最大扫测角度为 $140^{\circ}$ 。在水深 $30 \mathrm{~m}$ 的情况下, 通过 $3^{\sim} 4$ 次的反复扫测完全能够达到测点间距为 $3.6 \mathrm{~cm}$, 这样的测点密度完全能 够检测到水下细小的礁石, 能够非常详细的反映施工区域水下地形情况。

5.2 扫测水深精度

一次性的有效扫测宽度取决于扫杆的长度。扫杆在水流作用下会产生 形变, 入水深度越深, 变形越大, 有效扫测深度减少越多, 各情况下有效入 水深度减少情况。SeaBat 7125 型多波束测深系统的测量水深分辨率为 $6 \mathrm{~mm}$, 并且配备有精密的姿态仪, 能够实时获取精确的姿态数据并对测深数据进 行姿态改正, 测深数据不受水流影响而发生偏差, 能够保证扫测的有效扫 测深度。

5. 3 作业效率 
传统的硬式扫床方式需要先进行单波束水下地形测量进行初步检测, 未发现浅点的情况下再进行硬式扫床。而是扫床船通过控制缆绳的收放来 进行移动, 移动缓慢, 同时扫床过程中水位变化较大时, 还需要根据水位情 况调整扫杆的入水深度, 硬式扫床的作业效率非常低。采用多波束扫测系 统不需要先进行单波束水下地形测量, 可以直接进行扫床, 船速只要保持 在 $10 \mathrm{~km} / \mathrm{h}$ 以下即可, 施测过程中多波束系统测深数据根据GPS测量的水面 高实时进行水位补偿。作业效率比硬式扫床有明显提升。

5.4 效果展示

传统的硬式扫床方式是通过查看扫床轨迹图和扫杆情况来确认施工 效果, 成果展示手段单一, 直观性不足。多波束测深系统的测量数据在施测 过程中就可以实时显示水下区域的地形情况, 测量数据不仅可以制作平面 的地形图, 还可以制作三维模型, 展示的效果直观丰富。多波束系统和侧扫 声纳系统是海洋开发利用的重要手段, 两者在海洋调查、水下目标检测、 失事船舶扫描应用都很好。多波束声纳系统不仅可以绘制高分辨率的水下 地形图, 同时还可以利用背向散射强度地图声纳图像, 但分辨率较低的多 波束图像, 图像质量差, 随机干扰因素, 更直观, 易读性差, 无法比较。由于 既有互补, 又有外国学者长期以来两种系统结合了研究的可能性和优势, 取得了一定的效果, 我国的学者在两者的图像融合中也做了大量的工作。

\section{6 多波束与侧扫声呐数据的融合方法}

6. 1 等高线和等高线特征融合数据

校正后, 第一个拿起两个系统上的特征点 (轮廓特征点和灰度特征点) 为每个特征点MBES, SSS的位置误差范围内, 选择的特征点集, 然后根据相 关系数衡量来确定局部窗口的对应点, 其次之间的二次多项式作为图像变 形模型, 根据相同的地方来确定变形模型; 然后在计算残差的变形模型、 总误差、确定变形模型和SSS数据、图像重采样的过程中, 分别用二进制小 波对融合后的深度和强度进行重新采样。该方法要求扫描声纳系统具有较 深的探测功能。

\section{2冲浪特性的注册与整合}

在同一区域扫描和多波束声呐图像特征点的检测中, 从最近邻匹配点 得到的图像, 在图像间变换矩阵后, 利用空间变换完成配准, 采用加权融合 法实现积分。赵建湖等改进了算法, 并匹配算法, 最终实现了图像匹配。侯 雪等人通过对低分辨率图像进行采样, 获得更好的图像配准效果。此外, 改进了粗匹配算法在SURF算法中的距离测度函数, 提高了SURF算法的注册 速度。然后利用RANSAC算法, 实现了多波束和侧扫描声纳图像的精确配准。 最后, 对匹配图像进行小波变换融合。

\section{3 图像融合效果}

利用灰度均值、标准差、平均梯度、信息熵和综合评价指标, 对声纳 图像的融合效应进行评价, 如相互信息、信噪比、联合熵和相关系数。均 值和标准差反映了图像的平均亮度和均值的离散度。改进的质量、清晰度 和小细节对比度的平均梯度图像; 信息熵可以测量融合前后信息信息的变 化, 值越大, 融合图像所包含的信息越多。通过相互信息来衡量从原始图像 中获得多少信息; 信噪比、组合熵和相关系数测量图像的融合效果, 值越 大, 融合效果越好。
通过上述分析, 可以发现, 扫描声纳系统和多波束系统都是海底表面 的成像, 两者可以更好地获取海底信息。相比之下, 多波束声纳系统主要偏 重海底地形测量、定位精度的需求较高, 其数据处理精度的定位和深度测 量控制等, 和侧扫描声纳系统主要集中在降噪的声音和几何校正等。多波 束系统的一些传感器的误差从中央波束水深的影响增加梁的边缘, 即中央 梁边缘精度明显高于光束的准确性和侧扫描声纳在声发射信号在接收单 萍按照这样的安排, 不能真正反映水下图像, 直线距离校正通常无法消除 其影响。现有主要在遥感图像数据融合算法的基础上改进, 如在注册的过 程中会发生或多或少的准确性降低或分辨率降低的现象, 同时, 多波束声 纳系统和侧扫描声纳系统非常不同于遥感图像、遥感图像对于每个平面拼 接的, 但两个声纳系统是由平线组成的一个平面, 因为它的位移探针存在 空间, 每个萍不是在同一个平面上, 然后改变其梁射击方向也存在, 严格地 说, 这两个应该是一个三维图像, 因此使用数据融合仍存在一定的困难。

总之多波束声纳的计量测试需要大型基础设施的支撑, 用于水声基本 参数校准的六面消声水池和用于测深校准的原型深水港池都是必不可少 的。由于高昂的基建和维护费用, 大型消声水池主要用于军用声纳设备的 性能评价, 涉及民用海洋测量声纳的研究较少; 专门用于多波束声纳测深 性能计量校准的原型深水港池尚未建设, 以至于多波束声纳的测量溯源性 和可信度难以保证。多波束声纳计量检定或校准的核心是用计量标准对测 量仪器的计量性能进行评估, 以保证其测量的准确可靠。多波束声纳虽然 不是强制性检定测量仪器, 但是在海洋测绘、救助打捞、水运工程等领 域应用十分广泛, 其测量结果关系工程质量、航行安全等, 本测试系统的 建立, 主要从设备应用者角度出发, 为多波束声纳提供了一套测深和有 效条带宽度的测试装置和方法, 构成了一条不间断的比较链, 使国家计 量基准与水深探测工作计量器具联系起来, 为海洋科技发展提供准确可 溯源的测试结果。

\section{7 结束语}

多波束声呐系统一次发射接收声脉冲可以形成上百个波束, 即可获得 地理坐标系下的上百个水深数据, 极大地提高了水深测量的作业效率。因 此, 利用多波束声呐系统进行了测量, 并用传统扫床方式进行质量检验。这 对水深测量的质量控制具有非常重要的意义。

[参考文献]

[1]高君,肖付民,裴文斌,等.多波束测深精度检定方法[J].测绘科学技 术学报,2012,29(3):179-182.

[3]李素江,董江,裴文斌,等.RTK三维水深测量精度分析及试验验证 [J]。 海洋测绘.2012,(5):33-36.

[3] 程波, 蔡艳军, 蒋婷婷. 多波束与RTK三维水深测量技术的联合应用 [J].工程技术研究,2018,(4):11-17.

\section{作者简介：}

任皓(1991--), 男, 山东济南人, 汉族, 本科学历, 助理工程师, 研究方 向: 河道整治工程研究; 从事工作: 水文测量等相关工作。

张少飞(1990--),男,河北石家庄人,汉族,本科学历, 助理工程师,研 究方向：河道整治工程研究；从事工作：水文测量等相关工作。 